\title{
Reference assays for Clostridium difficile infection: one or two gold standards?
}

\author{
Timothy Planche, ${ }^{1,2}$ Mark Wilcox ${ }^{1,2}$
}

${ }^{1}$ Centre for Infection, Division of Cellular and Molecular

Medicine, St George's University of London, Cranmer Terrace, London, UK ${ }^{2}$ Microbiology, Leeds Teaching Hospitals and University of Leeds, Old Medical School, Leeds General Infirmary, Leeds, UK

\section{Correspondence to} Dr Timothy Planche, Centre for Infection, Division of Cellular and Molecular Medicine, St

George's University of London, Cranmer Terrace, London SW17 ORE, UK; tplanche@sgul.ac.uk

Accepted 19 October 2010 Published Online First 30 November 2010

\section{ABSTRACT}

Accurate diagnosis of Clostridium difficile infection (CDI) is essential for optimal treatment, prevention and control. There are two reference assays for $\mathrm{CDI}$ diagnosis: the cell cytotoxicity assay (CCTA) and toxigenic culture (TC). Importantly, these tests actually detect different targets: CCTA detects the presence of $C$ difficile toxins (primarily toxin $B$, but also toxin $A$ ), whereas TC detects the presence in the stool of $C$ difficile with the potential to produce toxin. Not surprisingly studies comparing the results of these assays show imperfect agreement. Thus, a faecal sample may be CCTA negative but TC positive, and this raises the crucial question about the clinical significance of the presence of $C$ difficile with the capacity to produce toxin but no actual detectable free toxin. A positive TC result indicates that a patient with diarrhoea is potentially infectious. TC also has the advantage that the cultured isolate is available for typing and for susceptibility testing. In general, however, CCTA has been shown to be a better test for the laboratory confirmation of CDI, although additional culture may be needed to optimise sensitivity. Crucially, when these reference assays are used to determine the accuracy of alternative diagnostic tests, care should be taken to compare methods with their appropriate standard (ie, compare tests that target equivalent end-points). Such issues have contributed to the variable and often suboptimal performance of rapid diagnostic tests for CDI. Further research is urgently needed to improve knowledge of the utility of routine diagnostic tests in CDI and the factors that influence their performance.

\section{INTRODUCTION}

Clostridium difficile infection (CDI) is a potentially life threatening, usually healthcare-associated infection that causes considerable morbidity and mortality. CDI has become more common in many countries during the last decade, and may be increasing in severity associated with the introduction of epidemic strains. Most infections are acquired in hospitals, where CDI frequently afflicts vulnerable patients already or recently receiving antibiotics. In the UK, national reporting of CDI is mandatory, and targets have been set to reduce the incidence of cases. Such enhanced surveillance has underpinned the decrease in reported cases seen in England between 2008 and 2009. ${ }^{1}$

Accurate diagnosis of CDI is vital for patient management and infection control. Correct diagnosis is also essential for the attainment of reliable surveillance data, and specifically to enable efficient tracking of infections, comparisons between institutions as part of performance management, and accurate determination of the efficacy of interventions to reduce CDI risk. False-negative test results may lead to patients not being treated appropriately for CDI, and these may not be appropriately isolated to reduce the risk of transmission. However, false-positive results can similarly have important consequences, including inappropriate cessation of antibiotics, unnecessary initiation of CDI treatment, and failure to investigate alternative diagnoses. In some instances patients who have false-positive results may be cohorted with true CDI cases (eg, on $C$ difficile wards), so potentially exposing these individuals to increased risk of acquiring a genuine infection. Furthermore, the assessment of the accuracy of tests to confirm the diagnosis of CDI depends critically on the choice and performance of a reference standard assay. Without the appropriate choice and performance of a reference assay to define true positives and true negatives, it is not possible to accurately develop and assess new tests. In turn, use of suboptimal tests cloud our understanding of the diagnosis, epidemiology and control of CDI, not least because we cannot reliably distinguish on clinical grounds between $C$ difficile and other infective or non-infective causes of diarrhoea.

\section{REFERENCE ASSAYS FOR C DIFFICILE}

The cell cytotoxicity assay (CCTA) has been traditionally regarded as the reference ('gold standard') assay for the laboratory confirmation of CDI. This assay relies on the detection of a cytopathic effect in cell culture that is neutralised by the presence of antibodies to $C$ difficile toxins. Cells (eg, Vero or Hep2 cells) are cultured in the presence of a faecal filtrate, with and without the presence of neutralising antitoxin antibodies. These cultures are examined microscopically at 24 and $48 \mathrm{~h}$ for evidence of a cytopathic effect (cell rounding due to apoptosis) that is prevented by the specific antitoxin. CCTA requires the ability to perform cell culture, which is being used much less frequently in laboratories as nucleic acid amplification tests are used more widely, and a degree of expertise to recognise a cytopathic effect. There are commercially available frozen cells, such as human foreskin fibroblasts (Diagnostic Hybrids, Athens, OH, USA). These cells are more convenient, but their use needs validation. Clearly, current CCTAs are relatively slow, although limited data suggest that positive results may be obtainable after shorter incubation periods ${ }^{2}$; nevertheless, in practice, results are only available from the day following sample submission.

Some authors consider that culture of $C$ difficile followed by a cell cytotoxicity (or possibly other 
toxin) assay of suspect colonies (toxigenic culture, TC) is a more sensitive test for CDI. ${ }^{3}{ }^{4} \mathrm{TC}$ relies on the anaerobic culture of $C$ difficile from faeces, usually preceded by alcohol shock of the faecal sample to remove vegetative bacteria (that may overgrow $C$ difficile), so selecting the hardy spores of $C$ difficile. The faecal sample is then cultured on specific agar plates (eg, $C$ difficile cefoxitin cycloserine egg-yolk, CCEY) for at least $48 \mathrm{~h}$, and typical colonies are identified. There are isolates of $C$ difficile that do not produce toxins, and so it is necessary to confirm that suspect isolates are actually toxigenic. Cytotoxigenic culture may therefore take 4-5 days to confirm the presence of a toxigenic isolate, making this somewhat impractical for routine diagnostic use. A variant approach involves testing colonies directly for evidence of toxin production using an immunoassay, which (although not validated for such use) can produce a positive TC result $1-2$ days after the sample is received. ${ }^{3} 5$ Also, similar to criticisms of CCTA, TC requires technical expertise to culture and identify $C$ difficile reliably. There are advantages associated with culture, ${ }^{3}$ including the ability to type isolates and to perform antibiotic susceptibility testing.

A number of studies have compared the performance of CCTA and TC (table 1). It is clear from these that, in general, TC detects more positive samples than the CCTA; CCTA has a sensitivity of about $75-85 \%^{6-13}$ compared with TC. These studies also showed that a variable proportion of cases $(2-15 \%)^{6-13}$ are TC negative, but positive by CCTA. Such observations may be due to the lack of sensitivity of culture methods, and this is exacerbated by the variable performance of $C$ difficile selective media. ${ }^{14}$ Notably, the levels of agreement between CCTA and TC vary greatly between test sites. It is therefore probable that there are key factors that affect the performances of the reference assays. For example, sample freshness, patient pretreatment with metronidazole or vancomycin, the timing of the assay in relation to symptom onset, or testing all faecal samples as opposed to only those in patients with clear evidence of diarrhoea ( $\geq 3$ unformed stools in $24 \mathrm{~h}$ ) may influence test results. As these factors vary greatly between sites and over time, and the proportion of discordant results may change, making it impossible to generalise from the results of individual studies.

The fact that TC is more often positive than CCTA, along with perceived technical difficulties in performing CCTA, has led some to claim that the former assay is the 'real gold standard' ${ }^{4}$ especially for determining the accuracy of alternative diagnostic tests for CDI. There is some irony to this debate considering that in many diagnostic laboratories neither of the references assays is used for the routine diagnosis of CDI. Crucially also, available diagnostic tests for CDI actually detect different targets (table 2 and discussed below). CCTA detects the presence of $C$ difficile toxins (primarily toxin $B$, but also toxin A), whereas TC detects $C$ difficile bacteria or spores that have the potential to produce toxin. Thus, a faecal sample may be CCTA negative but TC positive, and this raises the crucial question about the clinical significance of the presence of $C$ difficile with the capacity to produce toxin but no actual detectable free toxin. As toxin-producing $C$ difficile can be cultured from about $2 \%$ of the general population and $7-25 \%$ of hospitalised patients, a TC-positive result may occur in the absence of CDI (see below). Also, not all $C$ difficile strains produce the major toxins ( $A$ and $B$ ) that mediate disease. Not surprisingly, therefore, studies comparing the results of culture, CCTA and TC tests show imperfect agreement.

The development of CDI is a multistage process and is summarised in figure 1. The patient first needs to be exposed to (usually the spores of) $C$ difficile, which usually occurs close to the onset of CDI, but occasionally may reflect longer term carriage. Second, there needs to be overgrowth of $C$ difficile, and this normally occurs after disruption of the normal bowel flora by antibiotic therapy. Finally, there needs to be toxin production that causes diarrhoea \pm colitis. $C$ difficile may be cultured from the faeces of up to $30 \%$ of inpatients without diarrhoea who have been in hospital for several weeks. ${ }^{15} 16$ It remains unclear whether a patient with $C$ difficile culture positive faeces is transiently carrying or actually colonised by the bacterium. Thus, the reference assay may be positive at different stages before and during the development of CDI.

\section{EPIDEMIOLOGY OF C DIFFICILE AND REFERENCE ASSAYS}

A full understanding of the epidemiology of $C$ difficile, particularly with regards to the detection of cytotoxin or culture of cytotoxigenic $C$ difficile, is essential to interpret the results of the reference assays. Since the early 1980s, there have been many studies where faeces have been cultured for cytotoxigenic $C$ difficile from individuals with and without diarrhoea.

Table 1 Studies comparing of 'gold standard' methods for C. difficile showing sensitivity, specificity and kappa values

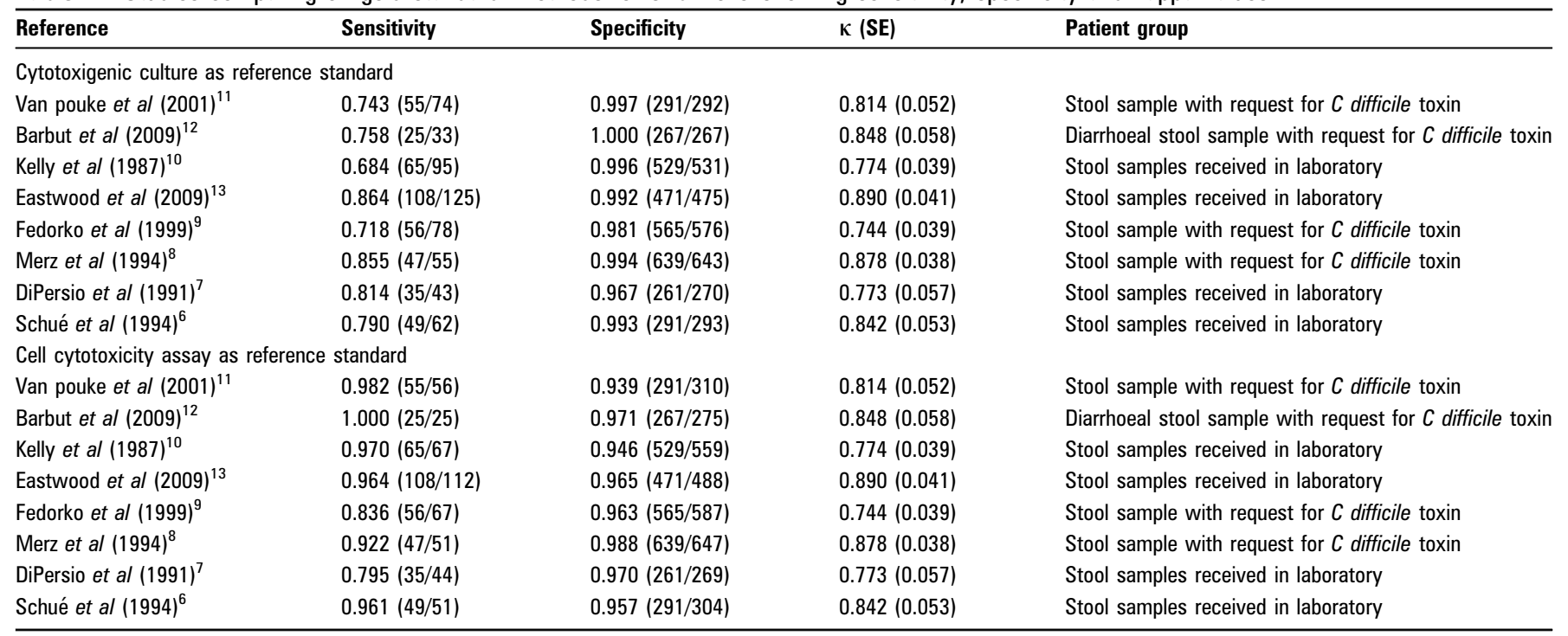


Table 2 Assays for Clostridium difficile

\begin{tabular}{|c|c|c|}
\hline Target & $\begin{array}{l}\text { Reference } \\
\text { method }\end{array}$ & Other methods \\
\hline$C$ difficile toxin(s) & $\begin{array}{l}\text { Cell-cytotoxicity } \\
\text { assay }\end{array}$ & $\begin{array}{l}\text { Toxin enzyme immunoassays; } \\
\text { toxin membrane assays }\end{array}$ \\
\hline C difficile & Culture & $\begin{array}{l}\text { Common antigen (glutamate } \\
\text { dehydrogenase) immunoassays }\end{array}$ \\
\hline $\begin{array}{l}\text { C difficile with the capacity } \\
\text { to produce toxin(s) }\end{array}$ & $\begin{array}{l}\text { Cytotoxigenic } \\
\text { culture }\end{array}$ & $\begin{array}{l}\text { Toxin gene PCR amplification } \\
\text { techniques }\end{array}$ \\
\hline
\end{tabular}

Cytotoxigenic C difficile was cultured from $2 \%(4 / 200)$ and $1.9 \%$ $(11 / 594)$ of faecal samples from healthy adults in $U^{17}$ and Swedish studies, ${ }^{18}$ none of whom had a positive cytotoxin assay. Studies of hospital inpatients typically show higher rates of detection of cytotoxigenic $C$ diffcile. A US study found $24 \%(192 / 810)$ of symptom-free admissions to have cytotoxigenic $C$ difficile cultured on rectal swabs. ${ }^{19}$ A UK study found $10 \%(29 / 284)$ of faecal samples from patients admitted to care of the elderly wards were $C$ difficile culture positive. A recent hospital admission is a known risk factor for the detection of $C$ difficile in faecal cultures. ${ }^{15} 16$

A number of studies have examined the rate of acquisition of $C$ difficile in patients during hospital admission. A US study found $C$ difficile in faecal cultures of $10 \%(65 / 678)$ patients admitted to the wards, ${ }^{16}$ and this increased to $18 \%(119 / 678)$ by the end of the study. A further US study found asymptomatic carriage of $C$ difficile in $7 \%(29 / 428)$ of admissions, ${ }^{20}$ and this increased to $20 \%$ (81/397) of asymptomatic patients, with a further 31 patients developing symptomatic CDI during the study. A study from Boston found an 11\% (45/406) rate of culture positivity for cytotoxigenic $C$ diffcile in new admissions, with a further $15 \%$ of initially culture-negative patients acquiring $C$ difficile during their hospital stay. ${ }^{15}$ Thus, the presence of cytotoxigenic $C$ difficile in the faeces of asymptomatic patients is relatively common. The rate of carriage is about $2 \%$ admissions are reported depending on the geographic setting of in the general population, ${ }^{17} 18$ but rates of $7-25 \%$ of hospital

the hospital. It should be noted that most of these studies are more than 10 years old, and that there may have been changes in the epidemiology of the carriage of $C$ difficile, notably since the introduction of newer strains and changes in hospital practice.

\section{CLINCIAL SIGNIFICANCE OF REFERENCE ASSAYS FOR C DIFFICILE}

It is clear from the above epidemiological data that the presence of $C$ difficile in faeces of asymptomatic patients may create diagnostic difficulties. If in-patients with a high rate of faecal carriage develop diarrhoea, there is a clear possibility that $C$ difficile culture will be positive regardless of the cause of the diarrhoea. A number of clinical studies have been designed to investigate the significance of reference assays in the laboratory confirmation of a diagnosis of CDI; these are discussed below.

A 1986 study $^{21}$ looked prospectively at the clinical picture of patients with CDI and compared them with controls. Cases were defined as adults with six unformed bowel motions in $36 \mathrm{~h}$ and either stool culture for $C$ difficile, positive toxin assay or endoscopic evidence of pseudomembranous colitis. Controls consisted of patients admitted to the same wards with a similar condition, but without diarrhoea. Patients with a positive culture as the only confirmation of CDI were analysed separately from the other cases. Over a year, 149 nine cases (109 positive by CCTA, and 40 that were only $C$ difficile culture positive) were compared with 148 controls. The 109 cases confirmed by CCTA had a longer hospital stay before diagnosis, higher rate of underlying disease and hypertension compared with controls. Cases also more commonly had fever and leucocytosis. In contrast, the 40 patients with only a positive culture result were clinically indistinguishable from controls, other than more frequently having fever. Colonoscopy was performed on 96 patients in the study, of whom 39 had evidence of pseudomembranous colitis (PMC). Of the patients with only a positive culture, $3 / 27$ (11\%) had endoscopic evidence of PMC. This contrasts with the patients with a positive CCTA of whom $35 / 68(51 \%)$ had evidence of PMC. From this it could be argued

Figure 1 Pathogenesis of Clostridium difficile infection and relationship with reference assays. Pos, positive; neg, negative.

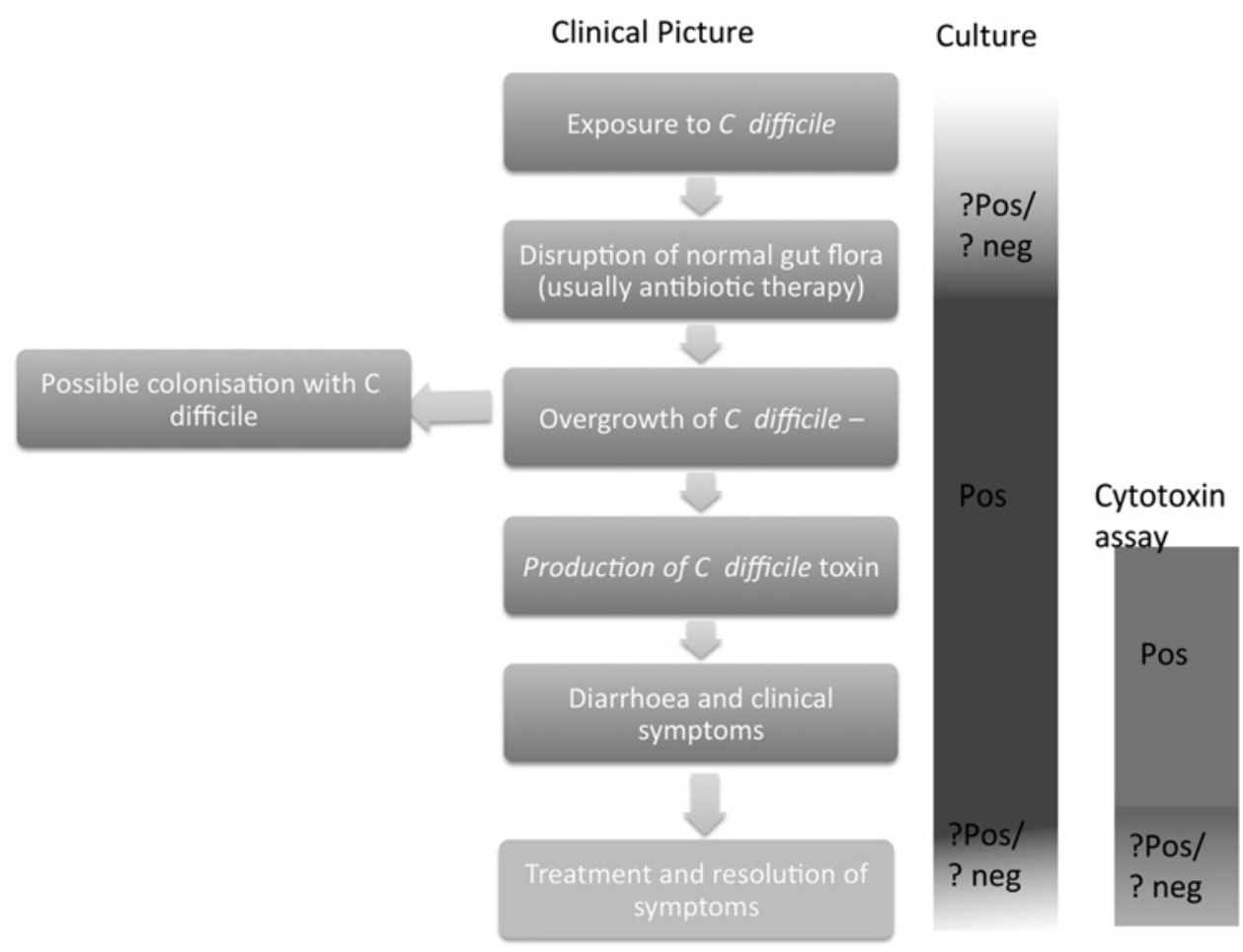


that only about $20 \%$ of diarrhoeal patients that are culture positive but CCTA negative may have CDI. It should be noted that of the 39 patients with PMC on endoscopy, 37/39 had positive culture result and $35 / 39$ had a positive CCTA, a difference that was not significant.

There are only a few studies comparing the results of endoscopy with those of reference assays. ${ }^{22-24}$ Initial studies from the early 1980s found CCTA to be positive in about $90 \%(27 / 30)$ of cases with PMC on endoscopy. ${ }^{23} 24$ In a recent UK study ${ }^{22}$ flexible sigmoidoscopy was carried out on 179 in-patients with diarrhoea ( $\geq 3$ watery stools in $24 \mathrm{~h}$ ) of unknown origin. Seventy-nine patients with known CDI were not included in the study. Faecal samples were taken at the time of sigmoidoscopy and the results of CCTA were compared with sigmoidoscopic appearance. PMC was present in 56 patients; of these CCTA was only positive in 27 (48\%) of cases. Frozen stool was available from only nine of the 29 CCTA-negative cases with PMC and all of these were TC positive. Though this study appears to show poor sensitivity of CCTA to detect PMC these data are difficult to interpret as patients with known CDI were excluded, patients had diarrhoea for a mean (SD) of 10 (1.2) days before sigmoidoscopy, and at least a third of the patients had received metronidazole before endoscopy. In addition, culture was not universally performed in all patients, and therefore a comparison between reference assays was not made. For these reasons, it is not possible to generalise from these results. While there may be improved sensitivity by performing culture as well as CCTA, further studies are needed to compare the results of endoscopy with laboratory reference assays in CDI. In practice, endoscopy is performed less often than formerly given concerns about its tolerability in typical frail older patients at risk of CDI.

A US study in 1986 looked at the clinical course in untreated patients that were CCTA negative but culture positive. ${ }^{25}$ Over 11 months, faecal samples were cultured from patients with diarrhoea and CCTA was performed. Physicians were notified of the result of the CCTA, but not of culture. Forty-five patients were included in the study, of whom 16 were culture positive and CCTA positive, and 29 were culture positive and CCTA negative (and outcome data were available for 22 of these). None of the patients that were CCTA negative received specific treatment for CDI. Seventeen of these 22 patients $(77 \%)$ recovered without treatment. Of the five patients who did not recover, one had a colectomy for inflammatory bowel disease (unrelated to CDI) and the other four died with ongoing diarrhoea, although it is uncertain whether the cause of death was related to CDI. Thirteen patients had further diagnostic studies (barium enema, colonoscopy or sigmoidoscopy) and none of these had any evidence of PMC; six had inflammatory bowel disease and seven had normal examinations.

A UK study in $1995^{26}$ examined the clinical picture of patients with faecal samples that were TC positive but negative by CCTA. Forty-one samples of 500 were CCTA positive, and nine samples from seven patients were culture positive but CCTA negative (a further two patients had non-cytotoxigenic $C$ difficle). A review of clinical records showed that three of these seven patients had carriage only, with the other four patients having possible disease. Two of those with possible disease had further investigations (colonoscopy or barium enema) that were negative and two further had negative repeat stool samples. The retrospective design of this study makes these estimates likely to overestimate the number of cases of possible disease.

Some other studies have examined the use of reference assays in $\mathrm{CDI}^{27}{ }^{28}$ However, these studies use laboratory results as part of the definition of CDI, and without clinical follow-up data or attribution of causality it is not possible to determine the true significance of test results. These studies generally indicate that patients who are culture positive but CCTA negative appear different from those who are CCTA positive. ${ }^{21}$ The great majority of cases do not appear to have $\mathrm{CDI}^{21} 26$ and most recover without specific treatment. ${ }^{25}$ Thus, while there is possibly a small increase in the sensitivity of detection of CDI if TC is used, such gains are likely to be at least offset by the poor specificity of culture-based diagnosis secondary to $C$ difficile carriage.

The available evidence is that the two reference assays for the detection of $C$ difficile are in fact answering two separate questions. The culture of cytotoxigenic $C$ difficile obviously indicates the presence of this organism in faeces, and thus indicates that this particular patient (notably with continuing diarrhoea) may pose a risk of cross-infection to others. However, given the relatively high rates of $C$ difficile carriage in hospitalised patients, and the results of detailed clinical studies, a positive culture or TC does not necessarily confirm that the cause of diarrhoea in a particular patient is CDI. In order to answer the question as to whether an individual patient with diarrhoea has CDI then it is necessary to perform a CCTA. It is an oversimplification to ask which of these reference methods is superior as they have different functions. If both reference tests were performed, a positive CCTA would confirm CDI, while negative tests for both would exclude CDI. Faecal samples negative by CCTA but positive by culture may be difficult to categorise, but it appears that the majority of these cases do not have CDI. Thus, clinical assessment of such cases would be important, with careful consideration of alternative diagnoses and reassessment if there is not prompt response to CDI therapy. It may prove impossible for a single reference standard to emerge for the laboratory diagnosis of $C$ difficile and a compound definition based on laboratory results and clinical findings will be developed.

\section{ASSESSMENT OF ACCURACY OF DIAGNOSTIC TESTS}

There is another important aspect of the use of CCTA and TC as reference assays that indirectly affects the routine laboratory diagnosis of CDI. While CCTA and TC are uncommonly used as frontline methods in diagnostic laboratories, routine tests for CDI are designed, assessed and optimised using these reference assays. It follows, therefore, that as the reference assays are not concordant, then the cut-off threshold used to define positive samples and the reported performance of laboratory tests will vary depending on the reference assay(s) used during development. The accuracy of laboratory diagnostic tests should be measured using reference assays that utilise the same or equivalent targets (see table 2). For example, $C$ difficile toxin enzyme immunoassays (EIAs) should be tested with CCTA as a reference standard; if they are measured against TC then accuracy will likely appear to be worse. In general, comparing toxin EIAs with TC will underestimate sensitivity, and comparing with PCR or glutamate dehydrogenase EIAs with CCTA will underestimate specificity. This effect is demonstrated in studies that report comparisons with both reference assays. ${ }^{13}$ There is also no clear rationale for using a different reference assay to attempt to resolve discrepant results in test and reference assays. As the reference assays measure different targets, then use of a second different reference assay on discrepant samples will not improve the assessment of the true accuracy of a diagnostic test. There has been a tendency for laboratories to stop performing the CCTA over recent years, but it is important to maintain the ability to perform this method in some laboratories, not least to be able to validate newer assays. 


\section{Take-home messages}

- Different reference methods produce different results for diagnosis of Clostridium difficile infection.

- Cytotoxigenic culture produces more positive results, but this does not necessarily imply a 'better' test.

- Clarity on the optimum reference (gold) standard method for the laboratory diagnosis of $C$ difficile infection requires much larger studies that include relevant clinical data.

- Selective use of the existing alternative reference (gold) standard methods can provide misleading performance results for new $C$ difficile detection methods.

Lack of consistency when investigating the accuracy of diagnostic tests for $\mathrm{CDI}$ has contributed to the poor performance of some methods. ${ }^{13} 29$ A further crucial issue here is the use during kit evaluations of samples containing a high proportions of positives relative to that seen in the typical diagnostic setting. This distorts (inflates) the positive predictive values of tests relative to those that are likely to be observed in practice. The positive predictive values of some diagnostic tests are unacceptably low, which will hinder clinical management of CDI and infection prevention and outbreak control, and makes epidemiological data unreliable. Thus, the suboptimal accuracy of routine tests, initially favoured for their convenience in comparison with CCTA and TC, has now led for calls to adopt two-stage methods for the routine laboratory diagnosis of $\mathrm{CDI}^{13} 2930$

\section{CONCLUSION}

In summary, results obtained from reference assays for the detection of CDI are not identical and answer different questions about $C$ difficile. TC identifies patients who are potentially infectious if they have diarrhoea. On available evidence, CCTA is a better test for the laboratory confirmation of CDI, although there needs to be confirmation of whether its sensitivity is improved with additional TC. Patients who are TC positive but CCTA negative, need careful clinical assessment. The majority of these patients do not have $\mathrm{CDI}^{21}{ }^{26}$ and improve without specific treatment. ${ }^{25}$ When using a reference assay to determine the accuracy of alternative diagnostic tests for CDI, it is important to use a method that targets the same end-point. Further research is urgently needed to improve knowledge of the diagnostic utility and relative performance characteristics of these tests and factors that influence their performance.

\section{Competing interests None.}

Provenance and peer review Not commissioned; externally peer reviewed.

\section{REFERENCES}

1. HPA and DH. Clostridium difficile Infection: How to Deal with the Problem. DH publications, 2008.

2. Settle CD, Wilcox MH. Comparison of the oxoid Clostridium difficile toxin a detection kit with cytotoxin detection by a cytopathic effect method examined at 4, 6, 24 and 48 h. Clin Microbiol Infect 1999;5:698-701.

3. Delmee M, Van Broeck J, Simon A, et al. Laboratory diagnosis of Clostridium difficile-associated diarrhoea: a plea for culture. J Med Microbiol 2005; 54:187-91.
4. Sloan LM, Duresko BJ, Gustafson DR, et al. Comparison of real-time PCR for detection of the tcdC gene with four toxin immunoassays and culture in diagnosis of Clostridium difficile infection. J Clin Microbiol 2008;46:1996-2001.

5. Thonnard J, Carreer F, Avesani V, et al. Toxin a detection on Clostridium difficile colonies from 24-h cultures. Clin Microbiol Infect 1996;2:50-4.

6. Schue V, Green GA, Monteil H. Comparison of the ToxA test with cytotoxicity assay and culture for the detection of Clostridium difficile-associated diarrhoea disease. J Med Microbiol 1994;41:316-18.

7. DiPersio JR, Varga FJ, Conwell DL, et al. Development of a rapid enzyme immunoassay for Clostridium difficile toxin a and its use in the diagnosis of $C$. difficile-associated disease. J Clin Microbiol 1991;29:2724-30.

8. Merz CS, Kramer C, Forman M, et al. Comparison of four commercially available rapid enzyme immunoassays with cytotoxin assay for detection of Clostridium difficile toxin(s) from stool specimens. J Clin Microbiol 1994;32:1142-7.

9. Fedorko DP, Engler HD, O'Shaughnessy EM, et al. Evaluation of two rapid assays for detection of Clostridium difficile toxin a in stool specimens. J Clin Microbiol 1999;37:3044-7.

10. Kelly MT, Champagne SG, Sherlock CH, et al. Commercial latex agglutination test for detection of Clostridium difficile-associated diarrhea. J Clin Microbiol 1987:25:1244-7.

11. Vanpoucke H, De Baere T, Claeys G, et al. Evaluation of six commercial assays for the rapid detection of Clostridium difficile toxin and/or antigen in stool specimens. Clin Microbiol Infect 2001;7:55-64.

12. Barbut F, Braun M, Burghoffer B, et al. Rapid detection of toxigenic strains of Clostridium difficile in diarrheal stools by real-time PCR. J Clin Microbiol 2009;47:1276-7.

13. Eastwood K, Else P, Charlett A, et al. Comparison of nine commercially available Clostridium difficile toxin detection assays, a real-time PCR assay for $C$. difficile $\mathrm{tcdB}$, and a glutamate dehydrogenase detection assay to cytotoxin testing and cytotoxigenic culture methods. J Clin Microbiol 2009;47:3211-17.

14. Brazier JS. The diagnosis of Clostridium difficile-associated disease. J Antimicrob Chemother 1998;41(Suppl C):29-40.

15. Samore MH, DeGirolami PC, Tlucko A, et al. Clostridium difficile colonization and diarrhea at a tertiary care hospital. Clin Infect Dis 1994;18:181-7.

16. Clabots CR, Johnson S, Olson MM, et al. Acquisition of Clostridium difficile by hospitalized patients: evidence for colonized new admissions as a source of infection. $J$ Infect Dis 1992;166:561-7.

17. Phillips KD, Rogers PA. Rapid detection and presumptive identification of Clostridium difficile by p-cresol production on a selective medium. J Clin Pathol 1981;34:642-4.

18. Aronsson B, Mollby R, Nord CE. Antimicrobial agents and Clostridium difficile in acute enteric disease: epidemiological data from Sweden, 1980-1982. J Infect Dis 1985; 151:476-81.

19. Shim JK, Johnson S, Samore MH, et al. Primary symptomless colonisation by Clostridium difficile and decreased risk of subsequent diarrhoea. Lancet 1998;351:633-6.

20. McFarland LV, Mulligan ME, Kwok RY, et al. Nosocomial acquisition of Clostridium difficile infection. N Engl J Med 1989;320:204-10.

21. Gerding DN, Olson MM, Peterson LR, et al. Clostridium difficile-associated diarrhea and colitis in adults. A prospective case-controlled epidemiologic study. Arch Intern Med 1986;146:95-100

22. Johal SS, Hammond J, Solomon K, et al. Clostridium difficile associated diarrhoea in hospitalised patients: onset in the community and hospital and role of flexible sigmoidoscopy. Gut 2004;53:673-7.

23. George WL, Rolfe RD, Harding GK, et al. Clostridium difficile and cytotoxin in feces of patients with antimicrobial agent-associated pseudomembranous colitis. Infection 1982;10:205-8.

24. George WL, Rolfe RD, Finegold SM. Clostridium difficile and its cytotoxin in feces of patients with antimicrobial agent-associated diarrhea and miscellaneous conditions. J Clin Microbiol 1982;15:1049-53.

25. Lashner BA, Todorczuk J, Sahm DF, et al. Clostridium difficile culture-positive toxin-negative diarrhea. Am J Gastroenterol 1986;81:940-3.

26. Bond F, Payne G, Borriello SP, et al. Usefulness of culture in the diagnosis of Clostridium difficile infection. Eur J Clin Microbiol Infect Dis 1995;14:223-6.

27. Riley TV, O’Neill GL, Bowman RA, et al. Clostridium difficile-associated diarrhoea: epidemiological data from Western Australia. Epidemiol Infect 1994;113:13-20.

28. Peterson LR, Olson MM, Shanholtzer CJ, et al. Results of a prospective, 18-month clinical evaluation of culture, cytotoxin testing, and culturette brand (CDT) latex testing in the diagnosis of Clostridium difficile-associated diarrhea. Diagn Microbiol Infect Dis 1988;10:85-91.

29. Planche T, Aghaizu A, Holliman R, et al. Diagnosis of Clostridium difficile infection by toxin detection kits: a systematic review. Lancet Infect Dis 2008;8:777-84.

30. Crobach MJ, Dekkers OM, Wilcox MH, et al. European society of clinical microbiology and infectious diseases (ESCMID): data review and recommendations for diagnosing Clostridium difficile-infection (CDI). Clin Microbiol Infect 2009;15:1053-66. 